\title{
Growth, Yield and Nutrient Status in Soil of Summer Fodder Maize (Zea mays L.) as Influenced by Residual Effect of INM and Direct Application of Varying Fertility Levels
}

\author{
H. A. Patel* and J. D. Thanki \\ Department of Agronomy, N. M. College of Agriculture, Navsari Agricultural University, \\ Navsari (Gujarat), India \\ *Corresponding author
}

\author{
A B S T R A C T
}

\section{Keywords}

Fodder maize, Integrated nutrient management,

Fertility levels, Residual effect

Article Info

Accepted:

22 October 2020

Available Online:

10 November 2020
A field experiment was conducted at the College Farm, Navsari Agricultural University, Navsari (Gujarat) during the rabi and summer seasons of 2018 and 2019to study the residual effect of integrated nutrient management treatments imposed in preceding chickpea on succeeding fodder maize crop. The treatments in rabi chickpea consisted of viz., $\mathrm{T}_{1}-100 \% \mathrm{RDF}\left(20 \mathrm{~N}+40 \mathrm{P}_{2} \mathrm{O}_{5}+00 \mathrm{~K}_{2} \mathrm{O} \mathrm{kg} / \mathrm{ha}\right), \mathrm{T}_{2}-75 \% \mathrm{RDF}, \mathrm{T}_{3}-100 \% \mathrm{RDF}+$ Rhizobium $+\mathrm{PSB}, \mathrm{T}_{4}-75 \% \mathrm{RDF}+$ Rhizobium $+\mathrm{PSB}, \mathrm{T}_{5}$ - control. These treatments were replicated four times in randomized block design. Insucceding summer season, each main plotwas splitted into four sub-plots for fodder maize with treatments consisting of varying fertility levels viz., $\mathrm{S}_{1}-100 \% \mathrm{RDF}\left(80 \mathrm{~N}+40 \mathrm{P}_{2} \mathrm{O}_{5}+00 \mathrm{~K}_{2} \mathrm{O} \mathrm{kg} / \mathrm{ha}\right), \mathrm{S}_{2}-75 \% \mathrm{RDF}, \mathrm{S}_{3}$ $50 \% \mathrm{RDF}$ and $\mathrm{S}_{4}-$ control to fodder maize resulting in twenty treatment combinations replicated four times in split plot design. Treatments $100 \% \mathrm{RDF}+$ Rhizobium $+\mathrm{PSB}\left(\mathrm{T}_{3}\right)$, $100 \% \mathrm{RDF}\left(\mathrm{T}_{1}\right)$ and $75 \% \mathrm{RDF}+$ Rhizobium $+\mathrm{PSB}\left(\mathrm{T}_{4}\right)$ showed significantly improvement in growth and yield attributes, yields and available nutrient status in soilin fodder maize. Among the four fertility levels directly applied in fodder maize, application of $100 \%$ RDF $\left(\mathrm{S}_{1}\right)$ significantly highest all growth and yield attributes, available nutrient status in soil.

\section{Introduction}

Maize (Zea mays L.) is an important cereal fodder crop. Maize ranks second in position after sorghum among the cereal fodder crops. It is the ideal fodder crop having quick growing habit, high yielding ability, palatability, nutritive value and acceptable to the cattle at any stage of growth. The maize is also most useful crop for making silage. It can be grown within still wider limits and tolerate minimum temperature of about $10{ }^{\circ} \mathrm{C}$ and maximum of $45{ }^{\circ} \mathrm{C}$.In Gujarat state, animals are mainly fed by poor quality roughages, straws of pearl millet, paddy and wheat. Thus, the state is not only facing the shortage of quantity but good quality fodder. The productivity of livestock is primarily a function of fodder intake. Therefore, the need is of two types i.e., to increase the production 
of fodder crops and to improve the quality of fodder.

In the recent past, cropping system approach has gained importance in agriculture and relative enterprises. A system consists of several components which are closely related to interacting among themselves. In agriculture, management practices are usually formulated for individual crops. However, farmers are cultivating different crops in different season based on their adaptability to a particular season, domestic needs and profitability, therefore production technology should be developed keeping in view all the crops grown in a year or more than one year if any sequence or rotation extends beyond one year. Such a package of management practices for all the crops leads to efficient use of costly inputs, besides reduction in production cost. For instants, residual effect of fertilizers applied and nitrogen fix by legumes can considerably bring down the production cost, if all the crops are considered when individual crops. In this context, cropping system approaches gaining importance.

\section{Materials and Methods}

The present investigation was carried out at the College Farm, Navsari Agricultural University, Navsari (Gujarat) during the summer and kharif seasons of 2018 and 2019 to study the residual effect of integrated nutrient management treatments imposed in preceding chickpea on succeeding fodder maize crop.

The soil of experimental field was clay in texture and low in organic carbon $(0.42 \%)$ and available nitrogen (196.80 kg/ha), medium in available phosphorus $(38.30$ $\mathrm{kg} / \mathrm{ha}$ ), high in available potassium (315.43 $\mathrm{kg} / \mathrm{ha})$ and slightly alkaline in reaction $(\mathrm{pH}$ 8.23). A total of six treatments were imposed in rabi chickpea viz., $\mathrm{T}_{1}-100 \% \mathrm{RDF}(20 \mathrm{~N}+$ $\left.40 \mathrm{P}_{2} \mathrm{O}_{5}+00 \mathrm{~K}_{2} \mathrm{O} \mathrm{kg} / \mathrm{ha}\right), \mathrm{T}_{2}-75 \% \mathrm{RDF}, \mathrm{T}_{3}-$ $100 \% \mathrm{RDF}+$ Rhizobium $+\mathrm{PSB}, \mathrm{T}_{4}-75 \%$ $\mathrm{RDF}+$ Rhizobium + PSB, $\mathrm{T}_{5}$ - control. These treatments were replicated four times in randomized block design. Then, in succeding summer season, each main plot treatment was splited into four sub plot treatments with four levels of recommended dose of fertilizers viz., $\mathrm{S}_{1}-100 \%$ RDF $\left(80 \mathrm{~N}+40 \mathrm{P}_{2} \mathrm{O}_{5}+00 \mathrm{~K}_{2} \mathrm{O}\right.$ $\mathrm{kg} / \mathrm{ha}), \mathrm{S}_{2}-75 \% \mathrm{RDF}, \mathrm{S}_{3}-50 \% \mathrm{RDF}$ and $\mathrm{S}_{4}-$ control to fodder maize resulting in twenty treatment combinations replicated four times in split plot design. The nitrogen was applied through urea $(46 \% \quad \mathrm{~N})$ and phosphorus through single superphosphate $\left(\begin{array}{lll}16 \% & \mathrm{P}_{2} \mathrm{O}_{5}\end{array}\right)$. The $50 \%$ dose of nitrogen and full dose of phosphorus were applied at the time of sowing, remaining 50\% dose of nitrogen was top dressed at 30 DAS. All the successive biometric observations during the crop growth were recorded periodically from these plants. The mean values of all observations were utilized for statistical analysis by using statistical procedures as described by Panse and Sukhatme (1967). The treatment effects on all the characters under study were compared by employing ' $F$ ' - test and the data was analysed in split plot design.

\section{Results and Discussion}

\section{Growth attributes}

Residual effect of treatments consisting of $100 \% \mathrm{RDF}+$ Rhizobium + PSB $\left(\mathrm{T}_{3}\right), 100 \%$ $\mathrm{RDF}\left(\mathrm{T}_{1}\right)$ and $75 \% \mathrm{RDF}+$ Rhizobium + $\operatorname{PSB}\left(\mathrm{T}_{4}\right)$ in chickpea proved to be significantly better than remaining treatments for almost all the growth attributes viz. plant height, number of leaves/plant and leaf: stem ratio at all the growth stages (Table 1). This might be due to the residual effect of the beneficial residual effect of addition of inorganic fertilizers along with biofertilizers and FYM under cropping sequence on growth 
attributes recorded by Malik (2003), Pankhaniya (2007), Samborlang et al., (2019).

Treatments with varying fertility levels in fodder maize, significantly highest plant height (Table 1) and number of leaves per plant was recorded under treatment of $100 \%$ $\operatorname{RDF}\left(\mathrm{S}_{1}\right)$ over $75 \% \operatorname{RDF}\left(\mathrm{S}_{2}\right), 50 \% \mathrm{RD}\left(\mathrm{S}_{3}\right) \mathrm{F}$ and control $\left(\mathrm{S}_{4}\right)$ at $30,50 \mathrm{DAS}$ and at harvest of fodder maize during first and second years and in pooled analysis. Significantly higher leaf: stem ratio was noted under treatment $100 \%$ RDF $\left(S_{1}\right)$ being at par with treatment $75 \% \operatorname{RDF}\left(\mathrm{S}_{2}\right)$ during individual years of study. While significantly highest leaf: stem ratio was recorded under the treatment $100 \%$ $\operatorname{RDF}\left(\mathrm{S}_{1}\right)$ during pooled analysis. Since, nitrogen is an integral part of chlorophyll helped in more photosynthesis and resulted in better growth. Adequate nitrogen fertilization of fodder maize influencing plant height, number of leaves per plant and photosynthetic efficiency. Appropriate phosphorus application might have helped in early root development and energy transfer in plant, which favoured better growth in fertilized plots. Similar findings were reported by Kumar et al., (2002), Kumar et al., (2016) and Jadhav et al., (2018).

\section{Yield attributes and yield}

Yield attributes (Table 2) of fodder maize crop viz., green fodder yield, dry matter content and dry fodder yield was significantly influenced due to residual effect of integrated nutrient management applied in rabi chickpea crop. Application of $100 \%$ RDF + Rhizobium + PSB $\left(\mathrm{T}_{3}\right)$ to preceding chickpea crop by producing significantly higher green fodder yield of fodder maize but remained at par with treatment $100 \% \operatorname{RDF}\left(\mathrm{T}_{1}\right)$ during first year only. While, significantly highest green fodder yield recorded under the treatment of $100 \% \mathrm{RDF}+$ Rhizobium + PSB $\left(\mathrm{T}_{3}\right)$ during second year of investigation and in pooled results. In first year, significantly higher dry matter content was recorded with application of $100 \% \mathrm{RDF}+$ Rhizobium + PSB $\left(\mathrm{T}_{3}\right)$ but it was at par with $100 \%$ RDF $\left(\mathrm{T}_{1}\right)$. However, highest dry matter content was obtained under the treatment $100 \%$ RDF + Rhizobium + PSB $\left(\mathrm{T}_{3}\right)$ during second year of study and in pooled analysis. Significantly higher dry fodder yield was noted under application of $100 \% \mathrm{RDF}+$ Rhizobium $+\mathrm{PSB}\left(\mathrm{T}_{3}\right)$ which was at par with $100 \%$ RDF $\left(\mathrm{T}_{1}\right)$ during first year. While, application of $100 \% \mathrm{RDF}+$ Rhizobium + PSB $\left(\mathrm{T}_{3}\right)$ recorded highest dry fodder yield during second year of study and in pooled analysis. The increased green and dry fodder yields of fodder maize due to integration of inorganic, common application of organic source i.e. FYM, along with biofertilizers (i.e. Rhizobium + PSB) application to preceding rabi chickpea reflected to good crop growth (growth attributes) resulted into influenced positively on yield and growth parameters might have positive correlation with green and dry fodder yields of fodder maize. Similar results were also reported by Pankhaniya (2007), Prajapat et al., (2014) and Dixit et al., (2015).

The effect of inorganic fertilizer on yield parameters like green fodder yield, dry matter content and dry fodder yield (Table 2) was found significant during both the years of experimentation and pooled analysis. The beneficial effect of higher fertilization on vegetative growth was reflected into increasing the fodder yield. Application of $100 \%$ RDF $\left(\mathrm{S}_{1}\right)$ produced significantly maximum green fodder yield, dry matter content and dry fodder yield over $75 \% \mathrm{RDF}$ $\left(\mathrm{S}_{2}\right), \quad 50 \%$ RDF $\left(\mathrm{S}_{3}\right)$ and control $\left(\mathrm{S}_{4}\right)$ treatments during 2018 and 2019 as well as in pooled analysis. Response of fertilizers depends on numerous variable factors such as environmental conditions, management systems and plant density. 
Table.1 Plant height, number of leaves/plant and leaf: stem ratio of fodder maize as influenced periodically by different treatments

\begin{tabular}{|c|c|c|c|c|c|c|c|c|c|}
\hline \multirow[t]{3}{*}{ Treatment } & \multicolumn{3}{|c|}{ Plant height (cm) } & \multirow{2}{*}{\multicolumn{3}{|c|}{ Number of leaves/plant }} & \multicolumn{3}{|c|}{ Leaf : stem ratio } \\
\hline & \multicolumn{3}{|c|}{ At harvest } & & & & \multicolumn{3}{|c|}{ At harvest } \\
\hline & 2018 & 2019 & Pooled & 2018 & 2019 & Pooled & 2018 & 2019 & Pooled \\
\hline \multicolumn{10}{|c|}{ I). Main plot treatments (rabi chickpea) } \\
\hline$T_{1}$ & 173.67 & 176.59 & 175.13 & 15.39 & 15.41 & 15.40 & 0.29 & 0.31 & 0.30 \\
\hline $\mathbf{T}_{2}$ & 169.78 & 170.96 & 170.37 & 14.96 & 14.71 & 14.84 & 0.27 & 0.28 & 0.28 \\
\hline $\mathbf{T}_{3}$ & 178.69 & 179.86 & 179.28 & 16.12 & 15.74 & 15.93 & 0.31 & 0.33 & 0.32 \\
\hline $\mathbf{T}_{4}$ & 170.94 & 172.22 & 171.58 & 15.04 & 14.86 & 14.95 & 0.28 & 0.29 & 0.29 \\
\hline $\mathbf{T}_{5}$ & 165.88 & 168.15 & 167.02 & 14.59 & 14.44 & 14.51 & 0.28 & 0.29 & 0.29 \\
\hline SEm \pm & 2.64 & 2.58 & 1.84 & 0.29 & 0.29 & 0.20 & 0.01 & 0.01 & 0.01 \\
\hline $\operatorname{CD}(\overline{\mathbf{P}}=\mathbf{0 . 0 5})$ & 8.13 & 7.95 & 5.38 & 0.88 & 0.89 & 0.59 & 0.02 & 0.03 & 0.02 \\
\hline CV (\%) & 6.14 & 5.94 & 6.04 & 7.54 & 7.65 & 7.60 & 10.29 & 12.28 & 11.38 \\
\hline \multicolumn{10}{|c|}{ II). Sub plot treatments (summer fodder maize) } \\
\hline$S_{1}$ & 181.25 & 183.04 & 182.15 & 16.73 & 16.55 & 16.64 & 0.32 & 0.33 & 0.33 \\
\hline $\mathbf{S}_{2}$ & 173.79 & 173.70 & 173.74 & 15.50 & 15.31 & 15.40 & 0.30 & 0.32 & 0.31 \\
\hline $\mathbf{S}_{3}$ & 168.22 & 170.30 & 169.26 & 14.59 & 14.59 & 14.59 & 0.28 & 0.30 & 0.29 \\
\hline $\mathbf{S}_{4}$ & 163.90 & 167.19 & 165.54 & 14.05 & 13.68 & 13.86 & 0.24 & 0.25 & 0.25 \\
\hline SEm \pm & 2.19 & 1.93 & 1.46 & 0.21 & 0.22 & 0.15 & 0.01 & 0.01 & 0.00 \\
\hline $\mathrm{CD}(\overline{\mathbf{P}}=\mathbf{0 . 0 5})$ & 6.24 & 5.51 & 4.50 & 0.61 & 0.63 & 0.47 & 0.02 & 0.02 & 0.01 \\
\hline CV $(\%)$ & 5.71 & 4.98 & 5.35 & 6.26 & 6.56 & 6.41 & 8.45 & 9.51 & 9.03 \\
\hline General mean & 171.79 & 173.56 & 172.67 & 15.22 & 15.03 & 15.12 & 0.29 & 0.30 & 0.29 \\
\hline \multicolumn{10}{|c|}{ Interaction $(\mathbf{M} \times \mathrm{S})$} \\
\hline SEm \pm & 4.90 & 4.32 & 3.27 & 0.48 & 0.49 & 0.34 & 0.01 & 0.01 & 0.01 \\
\hline $\mathrm{CD}(\overline{\mathbf{P}}=\mathbf{0 . 0 5})$ & NS & NS & NS & NS & NS & NS & NS & NS & NS \\
\hline $\begin{array}{l}\text { Interaction } \\
\text { (Pooled) }\end{array}$ & $\mathrm{SEm}+$ & \multicolumn{2}{|c|}{$\mathrm{CD}(\mathrm{P}=0.05)$} & $\mathrm{SEm} \pm$ & \multicolumn{2}{|c|}{$\mathrm{CD}(\mathrm{P}=0.05)$} & $\mathrm{SEm} \pm$ & \multicolumn{2}{|c|}{$\mathrm{CD}(\mathrm{P}=0.05)$} \\
\hline $\mathbf{M} \times \mathbf{Y}$ & 2.61 & \multicolumn{2}{|c|}{ NS } & 0.29 & \multicolumn{2}{|c|}{ NS } & 0.01 & \multicolumn{2}{|r|}{ NS } \\
\hline $\mathbf{S} \times \mathbf{Y}$ & 2.07 & \multicolumn{2}{|c|}{ NS } & 0.22 & \multicolumn{2}{|c|}{ NS } & 0.01 & \multicolumn{2}{|r|}{ NS } \\
\hline$M \times S \times Y$ & 4.62 & \multicolumn{2}{|c|}{ NS } & 0.48 & \multicolumn{2}{|c|}{ NS } & 0.01 & \multicolumn{2}{|c|}{ NS } \\
\hline
\end{tabular}


Table.2 Green fodder yield, dry matter content and dry fodder yield of fodder maize as influenced by different treatments

\begin{tabular}{|c|c|c|c|c|c|c|c|c|c|}
\hline \multirow[t]{2}{*}{ Treatment } & \multicolumn{3}{|c|}{ Green fodder yield (q/ha) } & \multicolumn{3}{|c|}{ Dry matter content $(\%)$} & \multicolumn{3}{|c|}{ Dry fodder yield (q/ha) } \\
\hline & 2018 & 2019 & Pooled & 2018 & 2019 & Pooled & 2018 & 2019 & Pooled \\
\hline \multicolumn{10}{|c|}{ I). Main plot treatments (rabi chickpea) } \\
\hline$T_{1}$ & 221.13 & 219.68 & 220.41 & 16.39 & 16.28 & 16.34 & 79.37 & 78.85 & 79.11 \\
\hline $\mathbf{T}_{2}$ & 213.40 & 210.43 & 211.92 & 15.58 & 15.65 & 15.62 & 76.59 & 75.53 & 76.06 \\
\hline $\mathbf{T}_{3}$ & 243.46 & 242.01 & 242.74 & 17.92 & 17.72 & 17.82 & 87.38 & 86.86 & 87.12 \\
\hline $\mathbf{T}_{4}$ & 218.37 & 217.22 & 217.79 & 15.97 & 15.99 & 15.98 & 78.38 & 77.96 & 78.17 \\
\hline $\mathbf{T}_{5}$ & 206.15 & 193.77 & 199.96 & 15.08 & 14.93 & 15.00 & 73.99 & 69.55 & 71.77 \\
\hline SEm \pm & 7.34 & 7.02 & 5.08 & 0.50 & 0.43 & 0.33 & 2.74 & 2.52 & 1.86 \\
\hline $\mathrm{CD}(\overline{\mathbf{P}}=0.05)$ & 22.63 & 21.64 & 14.83 & 1.54 & 1.34 & 0.97 & 8.44 & 7.77 & 5.43 \\
\hline $\mathrm{CV}(\%)$ & 13.32 & 12.97 & 13.15 & 12.35 & 10.76 & 11.58 & 13.85 & 12.97 & 13.42 \\
\hline \multicolumn{10}{|c|}{ II). Sub plot treatments (summer fodder maize) } \\
\hline$S_{1}$ & 256.27 & 256.98 & 256.62 & 18.96 & 18.61 & 18.78 & 91.98 & 92.24 & 92.11 \\
\hline $\mathbf{S}_{\mathbf{2}}$ & 228.16 & 219.66 & 223.91 & 16.58 & 16.34 & 16.46 & 81.89 & 78.84 & 80.37 \\
\hline $\mathbf{S}_{3}$ & 209.51 & 207.74 & 208.62 & 15.50 & 15.59 & 15.54 & 75.20 & 74.56 & 74.88 \\
\hline $\mathbf{S}_{4}$ & 188.06 & 182.12 & 185.09 & 13.72 & 13.91 & 13.82 & 67.50 & 65.37 & 66.43 \\
\hline SEm \pm & 4.76 & 6.06 & 3.85 & 0.42 & 0.37 & 0.28 & 2.10 & 2.11 & 1.49 \\
\hline $\mathrm{CD}(\overline{\mathbf{P}}=\mathbf{0 . 0 5})$ & 13.57 & 17.25 & 11.87 & 1.19 & 1.05 & 0.86 & 5.97 & 6.02 & 4.59 \\
\hline CV $(\%)$ & 9.66 & 12.50 & 11.15 & 11.57 & 10.19 & 10.91 & 11.84 & 12.16 & 12.00 \\
\hline General mean & 220.50 & 216.62 & 218.56 & 16.19 & 16.11 & 16.15 & 79.14 & 77.75 & 78.45 \\
\hline \multicolumn{10}{|c|}{ Interaction (M x S) } \\
\hline SEm \pm & 10.65 & 13.54 & 8.61 & 0.94 & 0.82 & 0.62 & 4.69 & 4.73 & 3.33 \\
\hline $\mathrm{CD}(\overline{\mathbf{P}}=\mathbf{0 . 0 5})$ & NS & NS & NS & NS & NS & NS & NS & NS & NS \\
\hline $\begin{array}{l}\text { Interaction } \\
\text { (Pooled) }\end{array}$ & $\mathrm{SEm} \pm$ & \multicolumn{2}{|c|}{$\mathrm{CD}(\mathrm{P}=0.05)$} & \multicolumn{2}{|c|}{$\mathrm{SEm} \pm$} & $\mathrm{CD}(\mathrm{P}=0.05)$ & \multicolumn{2}{|c|}{$\mathrm{SEm} \pm$} & $\mathrm{CD}(\mathrm{P}=0.05)$ \\
\hline $\mathbf{M} \times \mathbf{Y}$ & 7.19 & \multicolumn{2}{|c|}{ NS } & \multicolumn{2}{|c|}{0.47} & NS & \multicolumn{2}{|c|}{2.63} & NS \\
\hline $\mathbf{S} \times \mathbf{Y}$ & 5.45 & \multicolumn{2}{|c|}{ NS } & \multicolumn{2}{|c|}{0.39} & NS & \multicolumn{2}{|c|}{2.10} & NS \\
\hline$M \times S \times Y$ & 12.18 & \multicolumn{2}{|c|}{ NS } & \multicolumn{2}{|c|}{0.88} & NS & \multicolumn{2}{|c|}{4.70} & NS \\
\hline
\end{tabular}


Table.3 Available nutrient $\left(\mathrm{N}, \mathrm{P}_{2} \mathrm{O}_{5}\right.$ and $\left.\mathrm{K}_{2} \mathrm{O}\right)$ status of soil as influenced by different treatments after harvest of fodder maize

\begin{tabular}{|c|c|c|c|c|c|c|c|c|c|}
\hline \multirow[t]{2}{*}{ Treatment } & \multicolumn{3}{|c|}{ Available N (kg/ha) } & \multicolumn{3}{|c|}{ Available $\mathrm{P}_{2} \mathrm{O}_{5}(\mathrm{~kg} / \mathrm{ha})$} & \multicolumn{3}{|c|}{ Available $\mathrm{K}_{2} \mathrm{O}(\mathrm{kg} / \mathrm{ha})$} \\
\hline & 2018 & 2019 & Pooled & 2018 & 2019 & Pooled & 2018 & 2019 & Pooled \\
\hline \multicolumn{10}{|c|}{ I). Main plot treatments (rabi chickpea) } \\
\hline $\mathbf{T}_{1}$ & 207.89 & 210.00 & 208.95 & 48.83 & 49.39 & 49.11 & 326.45 & 322.96 & 324.70 \\
\hline $\mathbf{T}_{2}$ & 203.58 & 205.93 & 204.75 & 47.37 & 48.69 & 48.03 & 323.68 & 320.04 & 321.86 \\
\hline $\mathbf{T}_{3}$ & 208.81 & 214.79 & 211.80 & 50.36 & 52.09 & 51.22 & 328.23 & 325.92 & 327.08 \\
\hline $\mathbf{T}_{4}$ & 204.63 & 208.87 & 206.75 & 48.35 & 49.24 & 48.80 & 319.93 & 316.16 & 318.05 \\
\hline $\mathbf{T}_{5}$ & 201.96 & 202.94 & 202.45 & 47.06 & 47.91 & 47.49 & 317.51 & 312.88 & 315.19 \\
\hline SEm \pm & 1.60 & 1.77 & 1.19 & 0.62 & 0.70 & 0.47 & 6.22 & 5.92 & 4.30 \\
\hline $\mathrm{CD}(\overline{\mathbf{P}}=\mathbf{0 . 0 5})$ & 4.94 & 5.45 & 3.48 & 1.90 & 2.16 & 1.36 & NS & NS & NS \\
\hline $\mathrm{CV}(\%)$ & 3.12 & 3.39 & 3.26 & 5.09 & 5.68 & 5.40 & 7.70 & 7.41 & 7.56 \\
\hline \multicolumn{10}{|c|}{ II). Sub plot treatments (summer fodder maize) } \\
\hline $\mathbf{S}_{1}$ & 211.79 & 212.21 & 212.00 & 49.45 & 51.48 & 50.47 & 328.43 & 324.90 & 326.67 \\
\hline $\mathbf{S}_{2}$ & 208.75 & 210.56 & 209.65 & 49.52 & 49.54 & 49.53 & 325.39 & 321.19 & 323.29 \\
\hline $\mathbf{S}_{3}$ & 203.70 & 209.45 & 206.58 & 47.99 & 49.48 & 48.73 & 321.09 & 318.05 & 319.57 \\
\hline $\mathbf{S}_{4}$ & 197.25 & 201.81 & 199.53 & 46.63 & 47.35 & 46.99 & 317.74 & 314.22 & 315.98 \\
\hline SEm \pm & 1.42 & 1.31 & 0.97 & 0.45 & 0.51 & 0.34 & 3.96 & 4.04 & 2.83 \\
\hline $\mathrm{CD}(\overline{\mathrm{P}}=\mathbf{0 . 0 5})$ & 4.05 & 3.72 & 2.97 & 1.29 & 1.47 & 1.06 & NS & NS & NS \\
\hline CV $(\%)$ & 3.09 & 2.80 & 2.95 & 4.17 & 4.66 & 4.43 & 5.48 & 5.65 & 5.56 \\
\hline General mean & 205.37 & 208.51 & 206.94 & 48.40 & 49.46 & 48.93 & 323.16 & 319.59 & 321.38 \\
\hline \multicolumn{10}{|l|}{ Interaction $(\mathrm{M} \times \mathrm{S})$} \\
\hline SEm \pm & 3.18 & 2.92 & 2.16 & 1.01 & 1.15 & 0.77 & 8.85 & 9.03 & 6.32 \\
\hline $\mathrm{CD}(\overline{\mathbf{P}}=\mathbf{0 . 0 5})$ & NS & NS & NS & NS & NS & NS & NS & NS & NS \\
\hline Interaction (Pooled) & \multicolumn{2}{|c|}{$\mathrm{SEm} \pm$} & $\mathrm{CD}(\mathrm{P}=0.05)$ & \multicolumn{2}{|c|}{$\mathrm{SEm} \pm$} & $\mathrm{CD}(\mathrm{P}=0.05)$ & \multicolumn{2}{|l|}{ SEm \pm} & $\mathrm{CD}(\mathrm{P}=0.05)$ \\
\hline $\mathbf{M} \times \mathbf{Y}$ & \multicolumn{2}{|c|}{1.69} & NS & \multicolumn{2}{|c|}{0.66} & NS & \multicolumn{2}{|c|}{6.07} & NS \\
\hline $\mathbf{S} \times \mathbf{Y}$ & \multicolumn{2}{|c|}{1.37} & NS & \multicolumn{2}{|c|}{0.48} & NS & \multicolumn{2}{|l|}{4.00} & NS \\
\hline$M \times S \times Y$ & \multicolumn{2}{|c|}{3.05} & NS & \multicolumn{2}{|c|}{1.08} & NS & \multicolumn{2}{|l|}{8.94} & NS \\
\hline
\end{tabular}


The increase in green and dry fodder yield of fodder maize with increasing levels of fertilizers was due to remarkable improvement in yield attributes and yield like dry matter content, green and dry fodder yield as well as the residual effect of the INM treatments in preceding crop. The results are in conformity with those reported by Rathod et al., (2002), Kumar et al., (2004), Kumar et al., (2016) and Kumar et al., (2017).

\section{Nutrient status in soil}

Various INM treatments imposed in preceding crop had significant influence on available nitrogen, phosphorus and potassium in soil after harvest of summer fodder maize crop (Table 3) was found significant due to residual effect of different treatments applied to rabi chickpea but different treatments did not influence available potassium in soil during both years of experimentation. Application of $100 \% \mathrm{RDF}+$ Rhizobium + PSB $\left(\mathrm{T}_{3}\right)$ recorded significantly higher available nitrogen which was at par with $100 \% \mathrm{RDF}\left(\mathrm{T}_{1}\right)$ and $75 \% \mathrm{RDF}+$ Rhizobium + PSB $\left(\mathrm{T}_{4}\right)$ during first year. While during second year as well as in pooled analysis it was found at par with $\mathrm{T}_{1}$ only. Significantly higher available phosphorus in soil was registered under treatment $100 \% \mathrm{RDF}+$ Rhizobium + PSB $\left(\mathrm{T}_{3}\right)$ but it was found at par with treatment $100 \% \operatorname{RDF}\left(\mathrm{T}_{1}\right)$ during first year of study. Moreover, in second year and pooled data, significantly highest available phosphorus in soil was recorded with application of $100 \%$ RDF + Rhizobium + PSB $\left(\mathrm{T}_{3}\right)$. The total soil $\mathrm{N}$ content increased with the biofertilizer inoculation after the harvest of both chickpea and fodder maize crops. This was probably due to increased $\mathrm{N}$-fixation by Rhizobium + PSB inoculation. Biofertilizer inoculation also increased available $\mathrm{P}$ in soil due to the favourable effect on soil and increased solubilization of unavailable phosphorus by PSB. However, dual inoculation with Rhizobium + PSB had more pronounced influence on population of bacteria in rhizosphere. This might be due to the release of higher quantities of root exudates as soluble carbohydrates and sugars into rhizosphere. This, in turn, might have resulted in enhanced proliferation of bacteria added through inoculation. The increase in available $\mathrm{N}$ and $\mathrm{P}$ status of soil has also been reported by Jat and Ahlawat (2006) and Umale (2016).

Available nitrogen, phosphorus and potassium (Table 3) in soil after harvest of summer fodder maize crop was found significant due to different levels of RDF applied to fodder maize but different treatments did not influence available potassium in soil during both years and in pooled analysis. Application of $100 \% \operatorname{RDF}\left(S_{1}\right)$ was found significantly higher available nitrogen being at par with treatment $75 \%$ RDF $\left(\mathrm{S}_{2}\right)$ during both years and in pooled analysis. Whereas in second year, it was remained at par with $50 \%$ RDF $\left(\mathrm{S}_{3}\right)$. Significantly higher available phosphorus in soil after harvest of fodder maize crop was recorded with treatment $100 \% \operatorname{RDF}\left(S_{1}\right)$ which was at par with treatment $75 \%$ RDF $\left(\mathrm{S}_{2}\right)$ during first year of experimentation whereas in pooled data and during second year, treatment $100 \%$ RDF $\left(S_{1}\right)$ registered significantly highest available phosphorus in soil after harvest of fodder maize crop. The improvement in available phosphorus could be ascribed to addition of phosphorus through fertilizers. The present findings are in accordance with those reported by Kumar et al., (2017) and Patel et al., (2018).

Based on the above findings obtained from two years of experimentation, it can be concluded that for getting higher yield and maintenance of soil status, fodder maize was recorded due to residual effect of $100 \% \mathrm{RDF}$ $\left(20 \mathrm{~N}+40 \mathrm{P}_{2} \mathrm{O}_{5}+00 \mathrm{~K}_{2} \mathrm{O} \mathrm{kg} / \mathrm{ha}\right)+$ 
Rhizobium + PSB $\left(\mathrm{T}_{3}\right), 100 \% \mathrm{RDF}\left(\mathrm{T}_{1}\right)$ and summer fodder maize crop should be fertilized with $100 \%$ RDF $\left(80 \mathrm{~N}+40 \mathrm{P}_{2} \mathrm{O}_{5}+\right.$ $00 \mathrm{~K}_{2} \mathrm{O} \mathrm{kg} / \mathrm{ha}$ ) through inorganic fertilizer in chickpea- fodder maize cropping sequence in south Gujarat condition.

\section{References}

Dixit, A.K., Kumar, Sunil, Rai, A.K. and Kumar, T.K. 2015. System productivity, profitability, nutrient uptake and soil health under tillage, nutrient and weed management in rainfed chickpea- fodder sorghum cropping system. Indian Journal of Agronomy 60(2): 205-211.

Jadav, V.M., Patel, P.M., Chaudhari, J.B., Patel, J.M. and Chaudhari, P.P. 2018. Effect of integrated nutrient management on growth and yield of rabi forage maize (Zea mays L.). International Journal of Chemical studies 6(1): 2160-2163.

Jat, R.S. and Ahlawat, I.P.S. 2006. Direct and residual effect of vermicompost, biofertilizers and phosphorus on soil nutrient dynamics and productivity of chickpea- fodder maize sequence. Journal of Sustainable Agriculture28(1): 41-54.

Kumar, A., Thakur, K.S. and Munuja, S. 2002. Effect of fertility levels on promising hybrid maize (Zea mays) under rainfed condition of Himachal Pradesh. Indian Journal of Agronomy 47(4): 526-530.

Kumar, R., Singh, M., Meena, B.S., Kumar, S., Yadav, M.R., Parihar, C.M., Meena R.H. and Kumar, U. 2017. Quality characteristics and nutrient yield of fodder maize (Zea mays L.) as influenced by seeding density and nutrient levels in Indo-Gangetic plains. Indian Journal of Agricultural Sciences 87(9): 1203-1208.

Kumar, R., Singh, M., Tomar, S.K., Meena,
B.S. and D.K. Rathore, D.K. 2016. Productivity and nutritive parameters of fodder maize under varying plant density and fertility levels for improved animal productivity. Indian Journal of Animal Research 50(2): 199-202.

Malik, M.S. 2003. Effect of N, P, Rhizobium and PSB on rabi gram (Cicer arientinum L.) and their residual effect on succeeding kharif rice. Ph.D (Agri.) Thesis submitted to NAU, Navsari (Unpublished).

Pankhaniya, R.M. 2007. Integrated nutrient management in summer soybean [Glycine max. (L.) Merril]- kharif fodder sorghum [Sorghum bicolor (L.) Moench] sequence under South Gujarat conditions. Ph.D. thesis (unpublished) submitted to N.A.U., Navsari (Gujarat).

Patel, K.M., Patel, D.M., Gelot, D.G. and Patel, I.M. 2018. Effect of integrated nutrient management on green forage yield, quality and nutrient uptake of fodder sorghum (Sorghum bicolor L.). International Journal of Chemical Studies 6(1): 173-176.

Prajapat K., Vyas A.K. and Dhar, S. 2014. Productivity, profitability and land-use efficiency of soybean (Glycine max)based cropping systems under different nutrient management practices. Indian Journal of Agronomy59(2): 229-234.

Rathod, N.D., Meghani, M.N. and Dudhat, M.S. 2002. Response of forage sorghum (Sorghum bicolor) to different levels of nitrogen and phosphorus. Forage Research 28(1): 16-18.

Samborlang, K., Wanniang, Singh, A.K., Ram, V., Das, A., Lala, I. P., Ray, N. and Singh, J. 2019. Effect of organic and inorganic nutrient application in vegetable pea on growth, yield and net return from succeeding maize in vegetable pea- maize cropping sequence. Indian Journal of Hill Farming: 94-101. 
Umale, A. 2016. Influence of levels of nitrogen, phosphorus and biofertilizer on growth, yield attributes, yield and quality of rabi greengram (Vigna radiata $\mathrm{L}$.) and its residual effect on succeeding summer maize (zea mays L.) under middle Gujarat conditions. Ph.D. Thesis, Department of Agronomy, Anand Agricultural University, Anand, Gujarat.

\section{How to cite this article:}

Patel, H. A. and Thanki, J. D. 2020. Growth, Yield and Nutrient Status in Soil of Summer Fodder Maize (Zea mays L.) as Influenced by Residual Effect of INM and Direct Application of Varying Fertility Levels. Int.J.Curr.Microbiol.App.Sci. 9(11): 3186-3194.

doi: https://doi.org/10.20546/ijcmas.2020.911.384 